\title{
NOTA SOBRE O CULTIVO DO ARROZ (Oryza sativa L.), CV. IAC 47 E IAC 435 EM SOLUÇAO NUTRITIVA *
}

\author{
A.A. MEDEIROS ** \\ E. MALAVOLTA $* * *$
}

RESUMO

As soluções nutritivas de HOAGLAND \& ARNON (1950) no 2 de KARIM \& VLAMIS (1962) for am fornecidas a duas variedades de arroz, uma de sequeiro (IAC 47) e uma de irrigação (IAC 435). A primeira solução, que contém $N$ nítri co e $\mathrm{N}$ amoniacal, garantiu maior produção dé matéria seca e maior teor de $\mathrm{N}$ total nas plan tas que foram colhidas no meio do estádio dé perfilhamento. A aeração forçada mostrou - se desnecessária para o crescimento.

\section{INTRODUÇÃO}

A nutrição nitrogenada no arroz tem sido objeto de estudo, tanto com relação à fonte de nitrogênio quanto comres-

* Parte da Dissertação de Mestrado do 10 autor. Recebido pa ra publicação em 16/10/1980.

** EMBRAPA

*** Departamento de Química, E.S.A. "Luiz de Queiroz", e CENA, USP. 
peito à presença de oxigênio no meio (MALAVOLTA, 1957; VIETS, 1965; IMAI, 1977).

Para MENGEL \& KIRKBY (L978), a mais importante diferen ça entre a absorção do nitrato e amônia é a sensibilidade aó $\mathrm{pH}$. A melhor faixa do $\mathrm{pH}$ para a absorção da amônia é a próxí ma da neutralidade; o nitrato é mais rapidamente absorvido em pH baixo. Em arroz a absorção dos dois íons foi estudada por FRIED et alii (1963). Os autores concluíram que em pH alcali no a amônia foi absorvida em maior quantidade, enquanto dimi nuindo $\mathrm{o} \mathrm{pH}$ aumentava a absorção do nitrato mas mesmo em $\mathrm{p} \overline{\mathrm{H}}$ ácido, a absorção da amônia foi maior que a do nitrato.

KARIM \& VLAMIS (1962) admitem que a absorção da amônia em arroz, ocorra com mais rapidez que a do nitrato. Estes au tores observaram sintomas de toxidez em plantas de arroz cul= tivadas em solução nutritiva, cuja fonte de nitrogênio foi amônia. Relatam os autores que após três semanas do início da experimentação, na extremidade das folhas mais velhas apa receram queimaduras que expandiram para a base. No curto periodo de dois dias as folhas secavam e enrolavam.

MALAVOLTA (1954) concluiu que a redução no desenvolvimento das plantas alimentadas com amônia foi devida não ao acúmulo de grande quantidade da mesma mas a implicações nos mecanismos fotossintéticos e respiratório.

IMAI (1977), examinando diferentes níveis de amônia no crescimento e produção do arroz, relata que a concentração de $30 \mathrm{ppm}$ provoca efeito depressivo no crescimento das raízes e na produção. A aplicação de fósforo e a permuta, em parte, do amônia por nitrato, diminuiu o efeito. MENGEL \& VIRO (1978) não mencionam efeito negativo da amônia na concentração de $18 \mathrm{ppm}$ em pH 5,5. Neste nỉvel, o teor de amônia livre no tecido era baixo e a fração solúvel nitrogenada constituia-se de aminoácidos e amidas.

A forma amoniacal constitui a principal fonte de nitro gênio para o arroz irrigado (WELLS, 1962; SANCHEZ, 1975). $\bar{A}$ inundação que permanece durante algum tempo nos solos arrozeiros, produz modificações nas propriedades físicas, químicas e biológicas. A predominância da anaerobiose desfavorece 
a disponibilidade do nitrato na zona radicular devidoàs perdas pela desnitrificação e lixiviação (PATRICK \& MANAPATRA, 1968; MALAVOLTA, 1978).

PATRIK \& STURGIS (1955), estudando o efeito do oxigênio sobre a absorção do nitrato e amónia peloa arroz, concluỉram que não havia interação entre o fornecimento do oxigênio e absorção dois dois îns.

A incapacidade do arroz em reduzir o nitrato, foi admi tida por Bonner, citado por MALAVOLTA (1967), como sendo à causa do nitrato não ser eficiente na nutrição do arroz. Kel$1 y$, citado por PATRICK \& MAHAPATRA (1968), atribuiu as condições cloróticas das plantas de arroz no estádio nascediças que receberam nitrato como única fonte de nitrogênio à incapacidade de assimilar bem o nitrato e o efeito tóxico do mesmo. Ch' Wan Kwans, citado por KARIN \& VLAMIS (1962), cultivando arroz em solução nutritiva, observou uma clorose nas plantas cultivadas com nitrato semelhante à deficiência de ferro.

MALAVOLTA (1957) estudou a nutrição nitrogenada nas duas formas de nitrogênio, combinada e/ou isoladas, em várias condições: pH controlado e sem controle; com e sem arejamento; dois niveis de molibdênio. As conclusões são sumari zadas:

I. A acidez na solução amoniacal produz efeito menos prejudicial no desenvolvimento do arroz do que a alcalinização na solução nitrica;

II. Na solução com pH controlado não houve diferença estatistica no crescimento das plantas nas soluções com amônia ou com nitrato;

III. A aeração forçada exerceu efeito negativo no desenvolvimento das plantas;

IV. As plantas cultivadas no substrato com $\mathrm{pH}$ não con trolado, tiveram melhor desenvolvimento quando $\bar{o}$ nitrato e amonia foram fornecidos juntos na presença de molibdênio. 
MENGEL \& VIRO (1978) relatam que a absorção e assimila ção do nitrogênio nas plantas de arroz, depende do estadó energético. Em baixa intensidade de luz, plantas de arroz di minuîram a absorção de amônia; a absorção e assimilação dé nitrato foram porém mais afetadas. A incorporação do nitrogênio na fração insolúvel, proteỉna e ācido nucleico, foi maior nas plantas alimentadas com amônia que nas plantas com nitrato.

0 arroz tem uma conhecida capacidade de crescer em solos anaeróbicos (VLAMIS \& DAVIS, 1944). Nesta condição redutora há produção de substância tóxica aos vegetais oriundas do metabolismo anaeróbico dos microorganismos. ISHIZUCA (1971) ressalta, entretanto, a habilidade do arroz em oxidar o meio vizinho às raízes.

Para SHEIK (1973), a presença de óxido de fero revestindo a superfície radicular do arroz cultivado em solo com condição redutora, indica que nas proximidades das raízes prevalece ambiente oxidante. Segundo BARBER (1962), o arroz tem grande capacidade em transferir o oxigênio atmosférico para as raízes via colmo, por intermédio do tecido aerenquimático.

No presente trabalho, pretende-se comparar o efeito de duas soluções nutritivas, uma contendo $\mathrm{N}^{-\mathrm{NO}_{3}^{-}}$e $\mathrm{N}^{-\mathrm{NH}_{4}^{-}}$e outra possuindo somente $\mathrm{N}-\mathrm{NO}_{\overline{3}}^{-}$, no crescimento e absorção de $\mathrm{N}$ em presença e ausência de arejamento, em doiscultivares, uma de sequeiro e outra de irrigação.

MATERIAL E METODOS

Cultivares - foram empregados dois cultivares obtidos pelo Instituto Agronómico de Campinas, SP: IAC 47, de sequei ro e IAC 435, de irrigação.

Tratamentos - as soluções e tratamentos empregados encontram-se nas Tabelas 1 e 2 .

Condução - empregaram-se vasos de plástico de 1,51 de capacidade; durante 2 semanas as plantas receberam as solu- 
ções diluídas à metade e, a partir de então, metade das plan tas receberam solução diluída e o restante solução sem diluir.

Tabela 1 - Composição das soluções nutritivas de HOAGLAND \& ARNON n? 2 (1950) e KARIM \& VLAMIS (1962)

\begin{tabular}{|c|c|c|}
\hline Solução Estoque & $\mathrm{ml} / 1 *$ & $\mathrm{~m} 1 / 1 * *$ \\
\hline $\mathrm{KNO}_{3}$ & 4 & 1 \\
\hline $\mathrm{CaNO}_{3}$ & 6 & 1 \\
\hline $\mathrm{KH}_{2} \mathrm{PO}_{4}$ & - & 2 \\
\hline $\mathrm{MgSO}_{4}$ & 2 & 1 \\
\hline $\mathrm{NH}_{4} \mathrm{H}_{2} \mathrm{PO}_{4}$ & 1 & - \\
\hline solução $a * * *$ & 1 & - \\
\hline solução b**** & - & 1 \\
\hline $\mathrm{Fe}-\mathrm{EDTA}$ & 1 & 1 \\
\hline
\end{tabular}

* Solução hOAGLAND \& ARNON n! 2 (1950)

$* *$ Solução KARIN \& VLAMIS (1962)

*** Composição: $\mathrm{H}_{3} \mathrm{BO}_{3} 2,86 \mathrm{~g} / 1 ; \mathrm{MnCl}_{2} \cdot 4 \mathrm{H}_{2} \mathrm{O} 1,81 \mathrm{~g} / 1 ; \mathrm{ZnSO}_{4}$. $7 \mathrm{H}_{2} \mathrm{O} 0,22 \mathrm{~g} / 1 \cdot \mathrm{CuSO}_{4} \cdot 5 \mathrm{H}_{2} \mathrm{O} 0,08 \mathrm{~g} / 1 ; \mathrm{H}_{2} \mathrm{MOO}_{4}$. $\mathrm{H}_{2} \mathrm{O} \quad 0,02 \mathrm{~g} / 1$.

***** Composição: $\mathrm{KCl} 3,7 \mathrm{~g} / \mathrm{I} ; \mathrm{H}_{3} \mathrm{BO}_{3} 1,6 \mathrm{~g} / 1 ; \mathrm{MnSO}_{4} \cdot \mathrm{H}_{2} \mathrm{O} 0,9$ $\mathrm{g} / 1 ; \mathrm{ZnSO}_{4} \cdot 2 \mathrm{H}_{2} \mathrm{O} 0,6 \mathrm{~g} / 1 ; \mathrm{CuSO}_{4} \cdot 5 \mathrm{H}_{2} \mathrm{O} 0,13$ $\mathrm{g} / 1 ; \mathrm{H}_{2} \mathrm{MnO}_{4} 0,02 \mathrm{~g} / 1$.

Colheita e anälise - as plantas foram colhidas 40 dias depois da germinação no meio do período de perfilhamento; de pois de secas foram analisadas para $N$ total.

\section{RESULTADOS E DISCUSSÃO}

Comparando os dados das Tabelas 3 e 4, observa-se que as plantas cultivadas com nitrato, solução de KARIM \& VLAMIS 
Tabela 2 - Tratamentos e repetições (para cada cultivar)

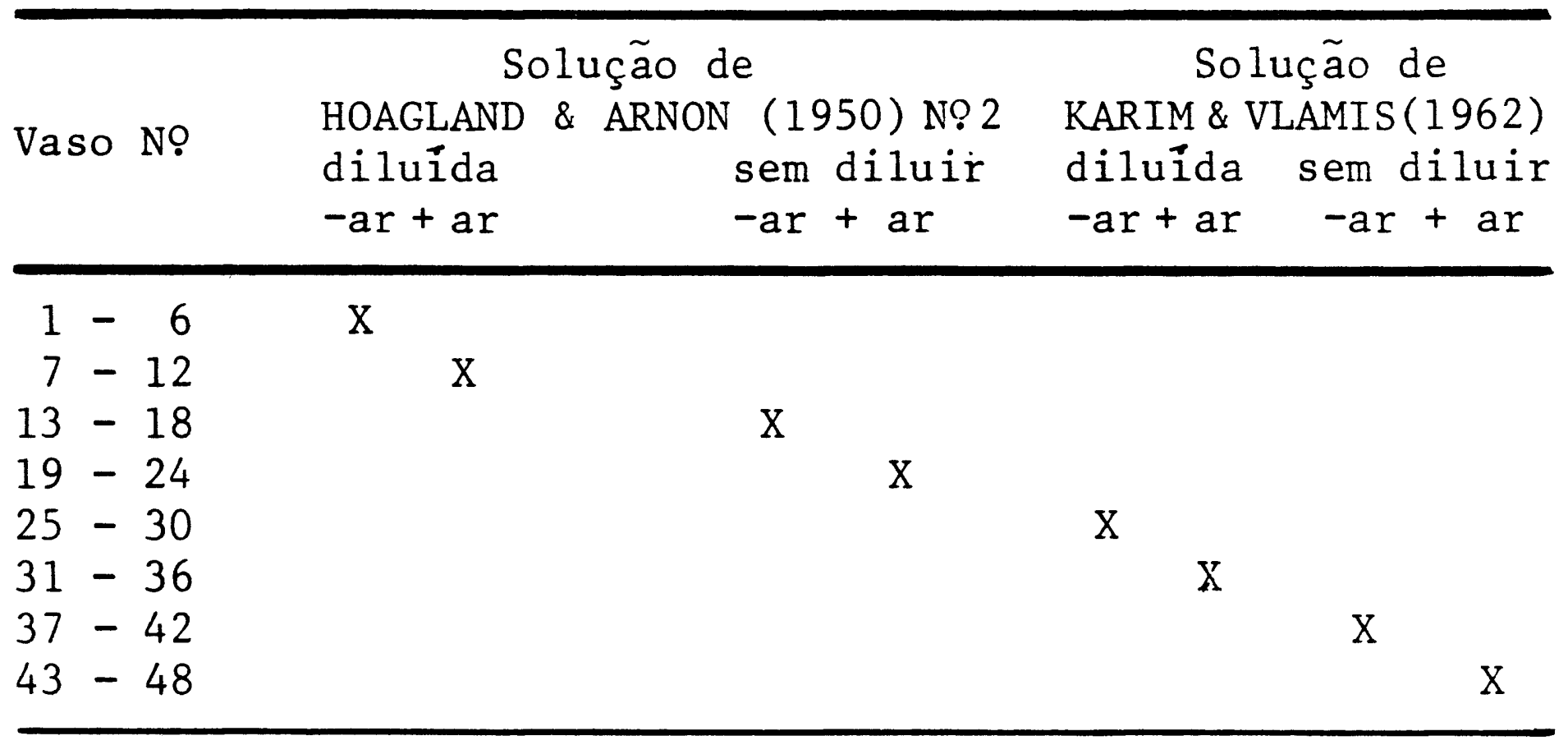

(1962), apresentaram menor produção de matéria seca e teor de nitrogênio, tanto na parte aérea como nas raizes. Na solução com nitrato as plantas, depois de poucos dias, mostravam-se menos vigorosas e as folhas emergentes eram cloróticas.

Kelly, citado por PATRICK \& MAHAiATKA (1968), observou em nascediças de arroz clorose nas plantas alimentadas com nitrato.

As plantas da solução contendo nitrato e amônia, ao con trário, apresentavam-se com uma cor verde e sem os sintomás descritos por KARIM \& VLAMIS (1962) e IMAI (1977) atribuíco à toxidez da amônia. A concentração desta solução era menor que as utilizadas pelos referidos autores o que deve explicar a diferença observada.

Nas Tabelas 3 e 4, observa-se que a concentração de nitrogênio nas plantas cultivadas com os dois ions foi superior a 4,0\%. Este nivel é próximo aos niveis ótimos relatados por ISHIZURA (1971) e MALAVOLTA (1978) para planta de arroz aos trinta dias de idade. Nas plantas com nitrato o teor de nitrogênio foi inferior a $3,5 \%$ na matéria seca. Pode-se 
Tabela 3 - Dados de crescimento dos dois cultivares de arroz, cultivados em solução de KARIM \& VLAMIS (1962) *

\begin{tabular}{|c|c|c|c|c|c|c|}
\hline \multirow{2}{*}{ Solução } & \multirow{2}{*}{$\begin{array}{c}\text { Parte aérea } \\
\text { g/planta }\end{array}$} & \multirow{2}{*}{$\begin{array}{c}\text { Raiz } \\
\text { g/plan } \\
\text { ta } \\
\end{array}$} & \multirow{2}{*}{$\begin{array}{c}\text { No de } \\
\text { perf } \underline{i} \\
\text { lhos } \\
\text { plantas }\end{array}$} & \multirow{2}{*}{$\begin{array}{l}\text { Raiz } \\
\mathrm{cm}\end{array}$} & \multicolumn{2}{|c|}{ N \% } \\
\hline & & & & & $\overline{\operatorname{Raiz}}$ & $\begin{array}{l}\text { Parte } \\
\text { aérea }\end{array}$ \\
\hline & Cultiva & I.A.C. & 435 & & & \\
\hline \multicolumn{7}{|l|}{ diluída } \\
\hline$+a r$ & 0,13 & 0,05 & 1,00 & 32,58 & 1,25 & 3,47 \\
\hline- ar & 0,12 & 0,05 & 0,80 & 30,33 & 1,21 & 3,05 \\
\hline \multicolumn{7}{|c|}{ sem diluir } \\
\hline+ ar & 0,16 & 0,06 & 1,08 & 32,16 & 1,40 & 3,32 \\
\hline - ar & 0,12 & 0,03 & 1,08 & 29,16 & 1,25 & 3,29 \\
\hline & Cultiva & I.A.C. & 47 & & & \\
\hline \multicolumn{7}{|l|}{ diluỉda } \\
\hline+ ar & 0,13 & 0,05 & 1,00 & 32,58 & 1,25 & 3,47 \\
\hline- ar & 0,12 & 0,05 & 0,83 & 30,33 & 1,20 & 3,44 \\
\hline \multicolumn{7}{|c|}{ sem diluir } \\
\hline+ ar & 0,16 & 0,06 & 1,08 & 32,16 & 1,40 & 3,37 \\
\hline- ar & 0,12 & 0,03 & 1,08 & 29,16 & 1,25 & 3,29 \\
\hline
\end{tabular}

* média de seis repetições

supor que, devido ao fato de ser a redutase do nitrato um en zimo de sintese induzida pelo substrato, as plantas recebendo a solução de KARIM \& VLAMIS (1962) eram deficientes em ni trogênio, o que é apoiado pela sintomatologia.

A maior produção de matéria seca nas plantas alimentadas com nitrato e amônia é explicado por COX \& REISENAUER (1962) pelo menor gasto de energia na utilização metabólica do nitrogênio.

Melhor explicação estaria na natureza da redutase 
Tabela 2 - Tratamentos e repetições (para cada cultivar)

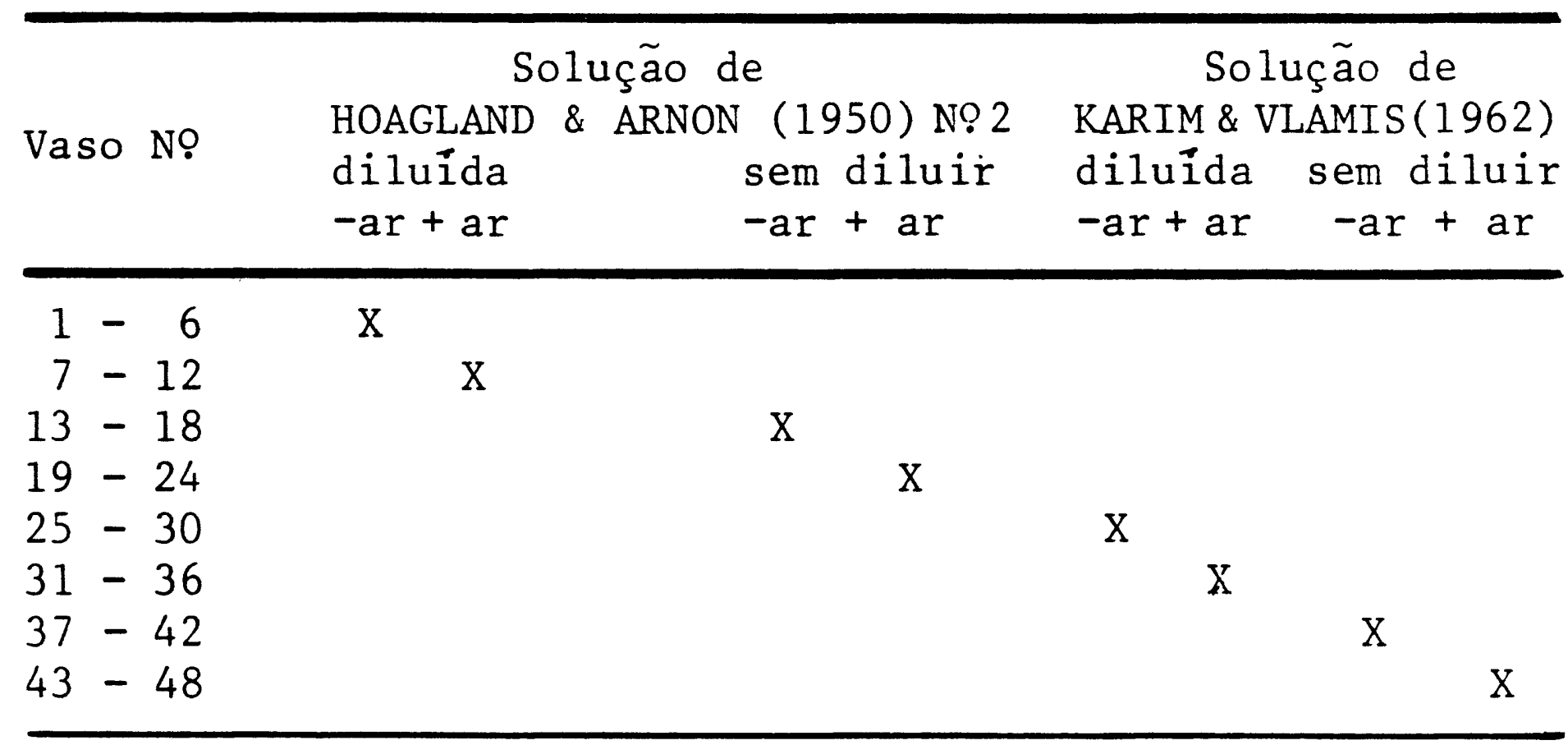

(1962), apresentaram menor produção de matéria seca e teor de nitrogênio, tanto na parte aérea como nas raízes. Na solução com nitrato as plantas, depois de poucos dias, mostravam-se menos vigorosas e as folhas emergentes eram cloróticas.

Kelly, citado por PATRICK \& MAHAiATKA (1968), observou em nascediças de arroz clorose nas plantas alimentadas com nitrato.

As plantas da solução contendo nitrato e amônia, ao con trário, apresentavam-se com uma cor verde e sem os sintomás descritos por KARIM \& VLAMIS (1962) e IMAI (1977) atrïbuído à toxidez da amônia. A concentração desta solução era menor que as utilizadas pelos referidos autores o que deve explicar a diferença observada.

Nas Tabelas 3 e 4, observa-se que a concentração de nitrogênio nas plantas cultivadas com os dois ions foi superior a 4,0\%. Este nivel é próximo aos niveis ótimos relatados por ISHIZURA (1971) e MALAVOLTA (1978) para planta de arroz aos trinta dias de idade. Nas plantas com nitrato o teor de nitrogênio foi inferior a $3,5 \%$ na matéria seca. Pode-se 


\section{CONCLUSÕES}

Cultivando-se dois cultivares de arroz em soluções nutritivas foi possível, atravēs das determinações de matéria seca acumulada até o meio do perfilhamento e da dosagem de $\mathrm{N}$ total, tirar as seguintes conclusões:

(1) o arejamento não aumentou o crescimento;

(2) o comportamento da variedade de sequeiro e da variedade de irrigação (IAC 45 e IAC 437, respectiva mente) foi o mesmo;

(3) a maior produção de matéria seca e o maior teor de $\Lambda$ total foram encontradas em plantas que receberam solução contendo $\mathrm{N}-\mathrm{NO}_{3}^{-}$e $\mathrm{N}^{-\mathrm{NH}_{4}^{-}}$.

\section{SUMMARY}

NOTE OIN THE GROWTH OF RICE PLANTS, CULTIVARS IAC 47 AND IAC 435, IN NUTRIENT SOLUTION

In a preliminary trial, a comparison was made of two nutrient solutions, HOAGLAND \& ARNON (1950) number 2, and KARIM \& VLAMIS (1962), both in the presence and in the absence of forced aeration. Plants grown during 40 days (till the middle of the tillering stage) showed a higher dry matter yield as well as a higher $\mathbb{N}$ content when receiving the first solution, which contains both nitrate and ammonium $N$, when compared with the latter whose nitrogen is only in the $\mathrm{NO}_{3}^{-}$form. Aeration was not necessary for maximum growth in either case.

\section{LITERATURA CITADA}

BARBER, D.A., 1962. The movement of ${ }^{15} 0$ though barley and rice plant. Jour. Exp. Bot. 13(39): 397-403. 
FRIED, M.; ZSOLDOS, F.; VOSE, P.B.; SHATOKHIN, I.L., 1963. Characterizing the $\mathrm{NO}_{3}$ and $\mathrm{NH}_{4}$ Uptake Process Rice Roots by Use of ${ }^{15} \mathrm{~N}$ Labelled $\mathrm{NH}_{4} \mathrm{NO}_{3}$. Physiologia Plantarum 18: $313-20$.

IMAI, H., 1977. The harmful effects of ammonium nitrogen on crop roots. In: The Society of the Science of Soil and Manure, Proceeding of the International Seminar in Soil Environment and Fetility Management in Intensive Agriculture, 634-40, Tokyo.

ISHIZUKA, Y., 1971. Physiology of the rice plant In Advances In Agronomy 23: 241-307, Academic Press, New York.

KARIM, A.Q.M.B.; VLAMIS, J., 1962. Micronutrient deficienty symptoms of rice grown in nutrient culture solutions. Plant Soil 3: 340-60.

MALAVOLTA, E., 1954. Studies on the nitrogen nutrition of rice. Plant Physiol. 29(1): 98-99.

MALAVOLTA, E., 1957. Contribuição ao estudo da alimentação nitrogenada do arroz (Oryza sativa L.), Piracicaba, ESALQ (Tese de Cátedra).

MALAVOLTA, E., 1978. Nutrição mineral e adubação do arroz irrigado, Ultrafertil, São Paulo, 68 p.

MENGEL, K.; VIRO, M., 1978. The significancy of plant energy status for the uptake and incorporation of $\mathrm{NH}_{4}^{+}$nitrogen by young rice plant. Soil Sci Plant Nutri. 24(3):407-416.

MENGEL, K.; KIRKBY, E.A., 1978. Principles of plant nutrition, International Potash Institute, Berna, $591 \mathrm{p}$.

PATRIC Jr., W.H.; STURGES, M.B., 1955. Concentration and movement of oxygen as related to absorption of ammonium and nitrate nitrogen by rice Soil Sci. Soc. Proc. 19(11): $59-62$.

PATRICK, Vn. H.; MAHAPATRA, I.C., 1968. Transformation and availability to rice of nitrogen and phosphorus and 
water 1logged Soil. Advances in Agronomy, Academic Press, New York, 20: 323-357.

SANCHEZ, P.A., 1975. Properties and management of Soil in the tropics, John Willey and Son, New York, 413-470.

SHEIKH, K.H., 1973. Effects of floadings and drainage and their alternation on the growth and uptake of nutrition by rice (oryza sativa L. indica var. I.R.8). Jour. Exp. Bot. $24(78): 66-75$.

VIETS, F.G., 1965. The plant need for and use of nitrogen. In: Soil Nitrogen, America Society of Agronomy, U.S.A., 508-548.

VLAMIS, J.; DAVIS, A.R., 1944. Effects of oxygen tension on certain physiological responses of rice, barley and tomato. Plant Physiol. 19: 35-51.

WELLS, J.P., 1962. Sources of nitrogen for rice, Agriculture Experimental Station, Report Series 115, University of Arkansas, 9 p. 
